$$
\text { "giering" — 2004/7/22 — 15:02 — page } 49 \text { — \#1 }
$$

\title{
Ein ungewöhnlicher Weg zu Jakob Steiners Umellipse eines Dreiecks und zur Steiner-Hypozykloide
}

\author{
OSWALd Giering \\ Herrn Professor József Szabó zum 65. Geburtstag gewidmet
}

\begin{abstract}
In real projective geometry of triangles two problems of collinear points are discussed. The problems differ only from the running through the vertices of a given triangle $A B C$. Resolving the problems we find two cubic curves $k_{S}$ and $k_{T}$. Affine specialization leads to the circumscribed Steiner ellipse about the triangle $A B C$ and shows us this ellipse in more general surroundings. Euclidean specialization leads to Steiners three-cusped hypocycloid.
\end{abstract}

Key words and phrases: projective geometry of triangles, plane cubics, circumscribed Steiner ellipse about a triangle, Steiners hypocycloid.

ZDM Subject Classification: G79, G75, G45.

In der Dreiecksgeometrie treten zahlreiche Kubiken (Kurven 3. Ordnung) auf, die sich einem gegebenen Dreieck $A B C$ als Ortskurven zuordnen lassen. Beispiele und Literaturhinweise zu diesem Themenkreis finden sich in $[1,2,3]$ und [6].

Im Folgenden werden zwei Kubiken $k_{S}$ und $k_{T}$ vorgestellt, die sich beim Studium von zwei projektivinvarianten Kollinearitätsproblemen einstellen. Beide Kubiken können in einen Kegelschnitt $k$ und eine Gerade zerfallen. Dabei ergeben sich Querverbindungen zum Satz von Pascal über die einem Kegelschnitt einbeschriebenen Sechsecke. Auch wird das von $k_{S}$ und $k_{T}$ aufgespannte Kubikenbüschel betrachtet. Nach affiner Spezialisierung bieten die Kubiken $k_{S}$ und

Copyright (C) 2004 by University of Debrecen 
$k_{T}$ die Möglichkeit, die einem Dreieck umbeschriebene Steiner-Ellipse ${ }^{1}$ in einem allgemeineren Zusammenhang zu sehen. Speziell am gleichseitigen Dreieck wird ein Hüllkurvenproblem behandelt, das zur Steiner-Hypozykloide ${ }^{1}$ führt.

\section{Ein Kollinearitätsproblem}

In der reellen projektiven Ebene sei ein festes Dreieck $A B C$ gegeben sowie auf jeder seiner Seiten $A B, B C, C A$ ein von $A, B, C$ verschiedener Punkt: $A^{\prime} \in B C$, $B^{\prime} \in C A, C^{\prime} \in A B$ (Abbildung 1). Die Punkte $A^{\prime}, B^{\prime}, C^{\prime}$ werden als Leitpunkte bezeichnet.

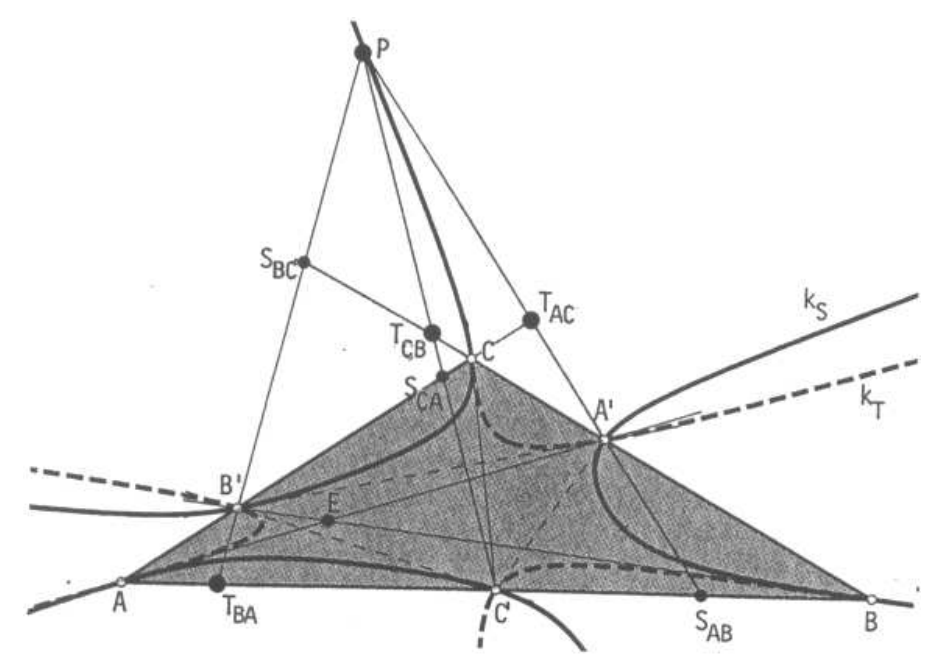

Abbildung 1

Wir betrachten nun die Verbindungsgeraden $P A^{\prime}, P B^{\prime}, P C^{\prime}$ eines beliebigen Punktes $P$ der Dreiecksebene mit den Leitpunkten $A^{\prime}, B^{\prime}, C^{\prime}$ sowie die (zweimal drei) Schnittpunkte:

$$
\begin{array}{lll}
S_{A B}:=P A^{\prime} \cap A B, & S_{B C}:=P B^{\prime} \cap B C, & S_{C A}:=P C^{\prime} \cap C A, \\
T_{A C}:=P A^{\prime} \cap A C, & T_{C B}:=P C^{\prime} \cap C B, & T_{B A}:=P B^{\prime} \cap B A .
\end{array}
$$

${ }^{1}$ Jakob Steiner (1796-1833), siehe etwa [5], S.637. 


$$
\text { "giering" — 2004/7/22 — 15:02 — page } 51 \text { — \#3 }
$$

Dem Punktetripel $\left(S_{A B}, S_{B C}, S_{C A}\right)$ liegt die Durchlaufung $A \rightarrow B \rightarrow C \rightarrow A$ des Dreiecks $A B C$ zugrunde, während das Punktetripel $\left(T_{A C}, T_{C B}, T_{B A}\right)$ der Durchlaufung $A \rightarrow C \rightarrow B \rightarrow A$ zugeordnet ist.

Wir fragen: Für welche Punkte $P$ liegen die drei Punkte $S_{A B}, S_{B C}, S_{C A}$ bzw. $T_{A C}, T_{C B}, T_{B A}$ kollinear?

\section{Das Kollinearitätsproblem in projektiver Sicht}

2.1. Zur Bearbeitung des in Abschnitt 1 formulierten Problems seien die Ecken des Dreiecks $A B C$ als Grundpunkte eines projektiven $x_{0} x_{1} x_{2}$-Koordinatensystems gewählt: $A(1,0,0), B(0,1,0), C(0,0,1)$. Die Dreiecksebene werde bei Bedarf komplex erweitert. Die Koordinaten der Leitpunkte $A^{\prime}, B^{\prime}, C^{\prime}$ seien:

$$
A^{\prime}\left(0, \alpha_{1}, \alpha_{2}\right), B^{\prime}\left(\beta_{0}, 0, \beta_{2}\right), C^{\prime}\left(\gamma_{0}, \gamma_{1}, 0\right) \quad \text { mit } \alpha_{1} \alpha_{2} \beta_{0} \beta_{2} \gamma_{0} \gamma_{1} \neq 0
$$

Der laufende Punkt $P$ erhalte die Koordinaten $\left(\xi_{0}, \xi_{1}, \xi_{2}\right)$. Dann besitzen die (viermal drei) Geraden $A B, B C, C A ; A^{\prime} B^{\prime}, B^{\prime} C^{\prime}, C^{\prime} A^{\prime} ; P A^{\prime}, P B^{\prime}, P C^{\prime} ; A A^{\prime}$, $B B^{\prime}, C C^{\prime}$ die Gleichungen:

$$
\begin{aligned}
& A B \ldots x_{2}=0, \quad B C \ldots x_{0}=0, \quad C A \ldots x_{1}=0 \\
& A^{\prime} B^{\prime} \ldots \alpha_{1} \beta_{2} x_{0}+\alpha_{2} \beta_{0} x_{1}-\alpha_{1} \beta_{0} x_{2}=0 \\
& B^{\prime} C^{\prime} \ldots \beta_{2} \gamma_{0} x_{1}+\beta_{0} \gamma_{1} x_{2}-\beta_{2} \gamma_{1} x_{0}=0 \\
& C^{\prime} A^{\prime} \ldots \gamma_{0} \alpha_{1} x_{2}+\gamma_{1} \alpha_{2} x_{0}-\gamma_{0} \alpha_{2} x_{1}=0 \\
& P A^{\prime} \ldots \xi_{0}\left(\alpha_{2} x_{1}-\alpha_{1} x_{2}\right)+\left(\alpha_{1} \xi_{2}-\alpha_{2} \xi_{1}\right) x_{0}=0 \\
& P B^{\prime} \ldots \xi_{1}\left(\beta_{0} x_{2}-\beta_{2} x_{0}\right)+\left(\beta_{2} \xi_{0}-\beta_{0} \xi_{2}\right) x_{1}=0 \\
& P C^{\prime} \ldots \xi_{2}\left(\gamma_{1} x_{0}-\gamma_{0} x_{1}\right)+\left(\gamma_{0} \xi_{1}-\gamma_{1} \xi_{0}\right) x_{2}=0 \\
& A A^{\prime} \ldots \alpha_{2} x_{1}-\alpha_{1} x_{2}=0 \\
& B B^{\prime} \ldots \beta_{0} x_{2}-\beta_{2} x_{0}=0 \\
& C C^{\prime} \ldots \gamma_{1} x_{0}-\gamma_{0} x_{1}=0
\end{aligned}
$$

Aus (1) und (3) berechnet man als Koordinaten der Punkte $S_{A B}, S_{B C}, \ldots, T_{C B}$ :

$$
\begin{array}{cc}
S_{A B}\left(\xi_{0} \alpha_{2}, \xi_{1} \alpha_{2}-\xi_{2} \alpha_{1}, 0\right), & T_{A C}\left(\xi_{0} \alpha_{1}, 0, \xi_{2} \alpha_{1}-\xi_{1} \alpha_{2}\right), \\
S_{B C}\left(0, \xi_{1} \beta_{0}, \xi_{2} \beta_{0}-\xi_{0} \beta_{2}\right), & T_{C B}\left(0, \xi_{1} \gamma_{0}-\xi_{0} \gamma_{1}, \xi_{2} \gamma_{0}\right), \\
S_{C A}\left(\xi_{0} \gamma_{1}-\xi_{1} \gamma_{0}, 0, \xi_{2} \gamma_{1}\right), & T_{B A}\left(\xi_{0} \beta_{2}-\xi_{2} \beta_{0}, \xi_{1} \beta_{2}, 0\right) .
\end{array}
$$




$$
\text { "giering" — 2004/7/22 — 15:02 — page 52 — \#4 }
$$

Die Punkte $S_{A B}, S_{B C}, S_{C A}$ liegen genau dann kollinear, wenn gilt:

$$
\begin{gathered}
\left|\begin{array}{ccc}
\xi_{0} \alpha_{2} & \xi_{1} \alpha_{2}-\xi_{2} \alpha_{1} & 0 \\
0 & \xi_{1} \beta_{0} & \xi_{2} \beta_{0}-\xi_{0} \beta_{2} \\
\xi_{0} \gamma_{1}-\xi_{1} \gamma_{0} & 0 & \xi_{2} \gamma_{1}
\end{array}\right|= \\
=\alpha_{2} \beta_{0} \gamma_{1} \xi_{0} \xi_{1} \xi_{2}+\left(\xi_{1} \alpha_{2}-\xi_{2} \alpha_{1}\right)\left(\xi_{2} \beta_{0}-\xi_{0} \beta_{2}\right)\left(\xi_{0} \gamma_{1}-\xi_{1} \gamma_{0}\right)=0
\end{gathered}
$$

Entsprechend liegen die Punkte $T_{A C}, T_{C B}, T_{B A}$ genau dann kollinear, wenn gilt:

$$
\begin{gathered}
\left|\begin{array}{ccc}
\xi_{0} \alpha_{1} & 0 & \xi_{2} \alpha_{1}-\xi_{1} \alpha_{2} \\
0 & \xi_{1} \gamma_{0}-\xi_{0} \gamma_{1} & \xi_{2} \gamma_{0} \\
\xi_{0} \beta_{2}-\xi_{2} \beta_{0} & \xi_{1} \beta_{0} & 0
\end{array}\right|= \\
=\alpha_{1} \beta_{2} \gamma_{0} \xi_{0} \xi_{1} \xi_{2}+\left(\xi_{2} \alpha_{1}-\xi_{1} \alpha_{2}\right)\left(\xi_{0} \beta_{2}-\xi_{2} \beta_{0}\right)\left(\xi_{1} \gamma_{0}-\xi_{0} \gamma_{1}\right)=0 .
\end{gathered}
$$

Wählt man (ohne Einschränkung) den Geradenschnittpunkt $A A^{\prime} \cap B B^{\prime}$ als Einheitspunkt $E(1,1,1)$ des projektiven Koordinatensystems, so folgt $\alpha_{1}=\alpha_{2}=$ $\beta_{0}=\beta_{2}=1$. Sind die Geraden $A A^{\prime}, B B^{\prime}, C C^{\prime}$ kopunktal in $E$, so folgt zusätzlich $\gamma_{0}=\gamma_{1}=1$.

Aus den Gleichungen (6) und (7) entnimmt man unter Beachtung der bisherigen Entwicklungen:

SAtz 1. Die Punkte $S_{A B}, S_{B C}, S_{C A}$ bzw. die Punkte $T_{A C}, T_{C B}, T_{B A}$ sind genau dann kollinear, wenn der laufende Punkt $P$ auf der Kubik $k_{S}$ mit Gleichung (6) bzw. auf der Kubik $k_{T}$ mit Gleichung (7) liegt (Abbildung 1).

Die Kubiken $k_{S}$ und $k_{T}$ gehen durch die Ecken der Dreiecks ABC sowie durch die Leitpunkte $A^{\prime}, B^{\prime}, C^{\prime}$. Man kennt somit 6 gemeinsame Punkte der Kubiken $k_{S}$ und $k_{T}$ (ihre Schnittpunkte mit den Dreieckseiten).

Die Geraden $A A^{\prime}, B B^{\prime}, C C^{\prime}$ schneiden die Kubiken $k_{S}$ und $k_{T}$ in $A, B, C$ jeweils zweifach und in $A^{\prime}, B^{\prime}, C^{\prime}$ jeweils einfach. Daher sind $A, B, C$ (zweifach zählend) und $A^{\prime}, B^{\prime}, C^{\prime}$ (einfach zählend) die - nach dem Satz von Bézout - 9 gemeinsamen Punkte der Kubiken $k_{S}$ und $k_{T}{ }^{2}$

\footnotetext{
${ }^{2}$ Man zeigt leicht, dass $A, B, C$ reguläre Punkte beider Kubiken sind. Folglich sind die Geraden $A A^{\prime}, B B^{\prime}, C C^{\prime}$ gemeinsame Tagenten der Kubiken. Eine Kubik ist durch 9 unabhähgige Punkte bestimmt (siehe etwa [4], S.201). Die 9 gemeinsamen Punkte $A, B, C$ (zweifach zählend) und $A^{\prime}, B^{\prime}, C^{\prime}$ sind wegen der Kollinearität der Punkte $S_{A B}, S_{B C}, S_{C A}$ bzw. $T_{A C}, T_{C B}, T_{B A}$ jedoch nicht unabhängig!
} 


$$
\text { "giering" — 2004/7/22 — 15:02 — page } 53 \text { - \#5 }
$$

Für $\alpha_{2} \beta_{0} \gamma_{1}+\alpha_{1} \beta_{2} \gamma_{0} \neq 0$ sind die Leitpunkte $A^{\prime}, B^{\prime}, C^{\prime}$ nicht kollinear (Abbildung 1). Dann gilt: In $A^{\prime}, B^{\prime}, C^{\prime}$ besitzt die Kubik $k_{S}$ jeweils die Tangente $A^{\prime} C^{\prime}, B^{\prime} A^{\prime}, C^{\prime} B^{\prime}$ und die Kubik $k_{T}$ jeweils die Tangente $A^{\prime} B^{\prime}, B^{\prime} C^{\prime}, C^{\prime} A^{\prime}{ }^{3}$

Für $\alpha_{2} \beta_{0} \gamma_{1}+\alpha_{1} \beta_{2} \gamma_{0}=0$ sind die Leitpunkte $A^{\prime}, B^{\prime}, C^{\prime}$ kollinear (Abbildung 2). Dann stimmen die Kubiken $k_{S}$ und $k_{T}$ überein und zerfallen in die Leitpunktgerade $A^{\prime} B^{\prime} C^{\prime}$ mit der Gleichung

$$
\alpha_{1} \beta_{2} x_{0}+\alpha_{2} \beta_{0} x_{1}-\alpha_{1} \beta_{0} x_{2}=0
$$

und den Kegelschnitt $k$ mit der Gleichung

$$
\beta_{2} \gamma_{0} x_{0} x_{1}-\beta_{0} \gamma_{0} x_{1} x_{2}+\beta_{0} \gamma_{1} x_{2} x_{0}=0 .
$$

Die den Kubiken $k_{S}$ und $k_{T}$ zugeordneten Punktetripel $\left(S_{A B}, S_{B C}, S_{C A}\right)$ und $\left(T_{A C}, T_{C B}, T_{B A}\right)$ sind dann kollinear.

Zerfallen die Kubiken $k_{S}$ und $k_{T}$ in die Leitpunktgerade $A^{\prime} B^{\prime} C^{\prime}$ und den Kegelschnitt $k$ und läuft der Punkt $P$ auf der Leitpunktgeraden $A^{\prime} B^{\prime} C^{\prime}$, dann sind die Punkte $S_{A B}, S_{B C}, S_{C A}$ sowie die Punkte $T_{A C}, T_{C B}, T_{B A}$ trivialerweise kollinear. Abbildung 2 zeigt $P$ als laufenden Punkt des Kegelschnitts $k$ und die drei jeweils kollinearen Punktetripel $\left(A^{\prime}, B^{\prime}, C^{\prime}\right),\left(S_{A B}, S_{B C}, S_{C A}\right),\left(T_{A C}, T_{C B}, T_{B A}\right)$.

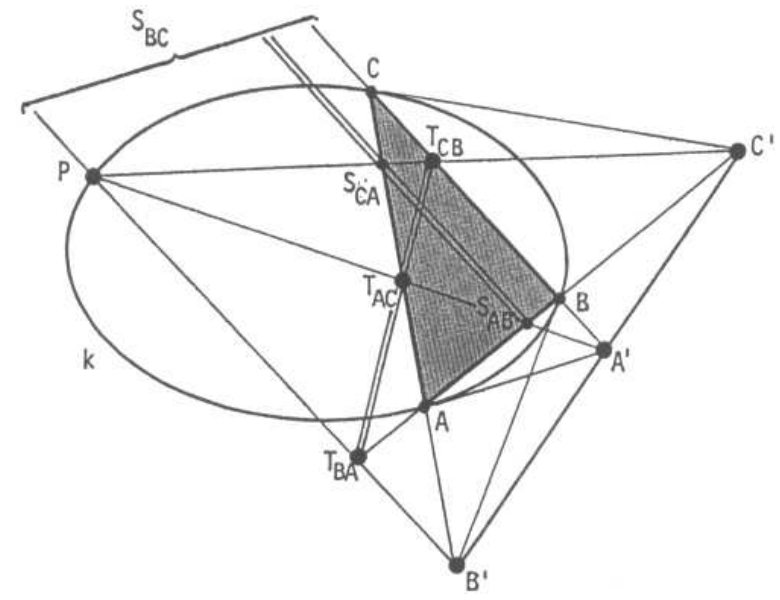

Abbildung 2

${ }^{3}$ Damit kennt man in algebraischer Zählung von jeder Kubiken $k_{S}$ und $k_{T} 12$ Punkte. 
Der Kegelschnitt $k$ besitzt die Eigenschaften:

(1) $k$ geht durch die Ecken des Dreiecks $A B C$.

(2) $k$ besitzt in $A$ die Tangente $A A^{\prime}$, in $B$ die Tangente $B B^{\prime}$ und in $C$ die Tangente $C C^{\prime}$. Damit ist das Dreieck $A B C$ dem Kegelschnitt $k$ derart einbeschrieben, dass die Schnittpunkte $A^{\prime}, B^{\prime}, C^{\prime}$ der Tangenten von $k$ in $A, B, C$ mit den jeweiligen Gegenseiten $B C, C A, A B$ kollinear liegen. Es zeigt sich also der folgende Spezialfall des Satzes von Pascal über ein dem Kegelschnitt $k$ einbeschriebenes Sechseck: Das Sechseck ist entartet in das Dreieck $A B C$ und die Kegelschnittangenten in $A, B, C$. Die Gegenseite von $A B, B C, C A$ ist jeweils die Tangente von $k$ in $C, A, B$.

(3) $k$ schneidet die Leitpunktgerade $A^{\prime} B^{\prime} C^{\prime}$ stets konjugiert komplex.

(4) Die Leitpunktgerade $A^{\prime} B^{\prime} C^{\prime}$ besitzt bezüglich $k$ den $\operatorname{Pol}\left(2 \alpha_{2} \beta_{0}, 2 \alpha_{1} \beta_{2},-\alpha_{2} \beta_{2}\right)$.

Bemerkung. Der Kegelschnitt $k$, der in den Punkten $A, B, C$ die Tangenten $A A^{\prime}, B B^{\prime}, C C^{\prime}$ besitzt, ist durch die 6 Elemente $A, B, C, A A^{\prime}, B B^{\prime}, C C^{\prime}$ überbestimmt. Man kann daher die folgende Schließungsaussage formulieren: Sind $A^{\prime} \in B C, B^{\prime} \in C A, C^{\prime} \in A B$ drei (von $A, B, C$ verschiedene) kollineare Punkte und ist $k$ der Umkegelschintt des Dreiecks $A B C$, der in $A$ die Tangente $A A^{\prime}$ und in $B$ die Tangente $B B^{\prime}$ besitzt, dann besitzt $k$ in $C$ die Tangente $C C^{\prime}$.

2.2. Fällt (entgegen unserer Voraussetzung) einer der Leitpunkte $A^{\prime}, B^{\prime}, C^{\prime}$ in eine Ecke des Dreiecks $A B C$, so zerfallen die Kubiken $k_{S}$ und $k_{T}$ ebenfalls (siehe (6) und (7)).

Für $A^{\prime}=C\left(\alpha_{1}=0, \alpha_{2}=1\right)$ sowie für $B^{\prime}=A\left(\beta_{2}=0, \beta_{0}=1\right)$ und $C^{\prime}=B\left(\gamma_{0}=0, \gamma_{1}=1\right)$ zerfällt $k_{S}$ in eine Dreieckseite $(A C, B A$, bzw. $C B)$ und einen Kegelschnitt $k^{*}$; die Kubik $k_{T}$ zerfällt in drei Geraden (für $A^{\prime}=C$ in $A C, C C^{\prime}, B B^{\prime}$, für $B^{\prime}=A$ in $B A, A A^{\prime}, C C^{\prime}$ und für $C^{\prime}=B$ in $\left.C B, B B^{\prime}, A A^{\prime}\right)$. Abbildung 3 zeigt den Fall $B^{\prime}=A$. Für $B^{\prime}=A$ geht der Kegelschnitt $k^{*}$ durch die Punkte $A, A^{\prime}, C$ und besitzt in $A^{\prime}$ die Tangente $A^{\prime} C^{\prime}$, in $C$ die Tangente $C C^{\prime}$; $k^{*}$ ist dadurch bestimmt.

Für $A^{\prime}=B\left(\alpha_{1}=1, \alpha_{2}=0\right)$ sowie für $B^{\prime}=C\left(\beta_{0}=0, \beta_{2}=1\right)$ und $C^{\prime}=A$ $\left(\gamma_{0}=1, \gamma_{1}=0\right)$ zerfällt die Kubik $k_{S}$ in drei Geraden (für $A^{\prime}=B$ in $A B, B B^{\prime}$, $C C^{\prime}$, für $B^{\prime}=C$ in $B C, C C^{\prime}, A A^{\prime}$ und für $C^{\prime}=A$ in $C A, A A^{\prime}, B B^{\prime}$ ); die Kubik $k_{T}$ zerfällt jeweils in eine Dreieckseite und einen Kegelschnitt.

Das Studium weiterer Zerfallsmöglichkeiten, die sich einstellen, wenn Leitpunkte mit Dreiecksecken inzidieren, überlassen wir dem Leser. 


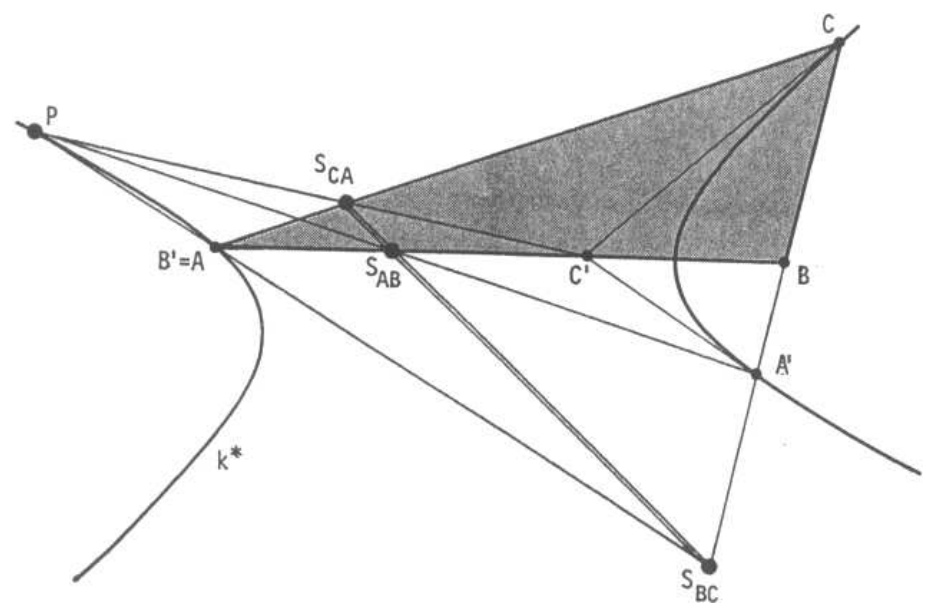

Abbildung 3

2.3. Das von den Kubiken $k_{S}$ und $k_{T}$ aufgespannte Kubikenbüschel wird unter Verwendung der projektiven Büschelparameter $\lambda, \mu$ beschrieben durch die Gleichung:

$$
\begin{aligned}
& \left(\lambda \alpha_{2} \beta_{0} \gamma_{1}+\mu \alpha_{1} \beta_{2} \gamma_{0}\right) \xi_{0} \xi_{1} \xi_{2}+ \\
& +(\lambda-\mu)\left(\xi_{1} \alpha_{2}-\xi_{2} \alpha_{1}\right)\left(\xi_{2} \beta_{0}-\xi_{0} \beta_{2}\right)\left(\xi_{0} \gamma_{1}-\xi_{1} \gamma_{0}\right)=0 .
\end{aligned}
$$

Aus (10) folgt unmittelbar:

SATz 2. Die Büschelkubik (10) zerfällt

für $\lambda-\mu \neq 0$ und $\lambda \alpha_{2} \beta_{0} \gamma_{1}+\mu \alpha_{1} \beta_{2} \gamma_{0}=0$ in die Geraden $A A^{\prime}, B B^{\prime}, C C^{\prime}$ und für $\lambda-\mu=0$ und $\alpha_{2} \beta_{0} \gamma_{1}+\alpha_{1} \beta_{2} \gamma_{0} \neq 0$ in die Dreieckseiten $A B, B C, C A$.

Für $\alpha_{2} \beta_{0} \gamma_{1}+\alpha_{1} \beta_{2} \gamma_{0}=0$ stimmen die Kubiken $k_{S}$ und $k_{T}$ nach Satz 1 überein und zerfallen in die Leitpunktgerade $A^{\prime} B^{\prime} C^{\prime}$ und den Kegelschnitt $k$.

Bemerkung. Die Geraden $\xi_{1}=\lambda \xi_{0}$ des Geradenbüschels mit Zentrum $C$ schneiden die Kubik $k_{S}$ in $C$ und in zwei weiteren Punkten, deren Koordinaten durch $\xi_{1}=\lambda \xi_{0}$ und eine quadratische Gleichung für $\xi_{2}: \xi_{0}$ bestimmt sind, die sich einstellt, wenn man $\xi_{1}=\lambda \xi_{0}$ in (6) einsetzt. Ist $F(\lambda)$ die Lösung dieser Gleichung, dann stellt $\xi_{1}=\lambda \xi_{0}, \xi_{2}=F(\lambda) \xi_{0}$ eine Parameterdarstellung von $k_{S}$ mit Parameter $\lambda$ dar. Entsprechendes gilt für $k_{T}$. 


\section{Das Kollinearitätsproblem in affiner und euklidischer Sicht}

3.1. Wird in der projektiven Ebene des Dreiecks $A B C$ eine Gerade als Ferngerade ausgezeichnet, dann besitzen die Kubiken $k_{S}, k_{T}$ eine oder drei Asymptoten, je nachdem die Ferngerade die Kubik in einem oder in drei verschiedenen reellen Punkten schneidet. Ist die Ferngerade Ferntangente oder Fernwendetangente der Kubik, dann fallen zwei bzw. drei reelle Schnittpunkte zusammen.

Ein interessanter affiner Sonderfall stellt sich ein, wenn alle drei Leitpunkte $A^{\prime}, B^{\prime}, C^{\prime}$ Fernpunkte sind. Dann gilt: $A B\left\|C C^{\prime}, B C\right\| A A^{\prime}, C A \| B B^{\prime}$. In diesem Fall stimmen die Kubiken $k_{S}$ und $k_{T}$ nach Satz 1 überein und zerfallen in die Leitpunktgerade $A^{\prime} B^{\prime} C^{\prime}$ und einen Kegelschnitt $k$. Der Kegelschnitt $k$ ist in dieser Interpretation notwendig eine Ellipse, da die Leitpunktgerade $A^{\prime} B^{\prime} C^{\prime}$ nach Abschnitt 2 den Kegelschnitt $k$ stets konjugiert komplex schneidet. Daraus folgt (Abbildung 4):

Satz 3. Sind in Satz 1 die Leitpunkte $A^{\prime}, B^{\prime}, C^{\prime}$ eines Dreiecks $A B C$ kollinear und wird die Leitpunktgerade $A^{\prime} B^{\prime} C^{\prime}$ als Ferngerade interpretiert, dann ist der Kegelschnitt $k$ die dem Dreieck $A B C$ umbeschriebene Steiner-Ellipse (jene dem Dreieck $A B C$ umbeschriebene Ellipse, deren Tangenten in den Ecken zu den Gegenseiten parallel sind).

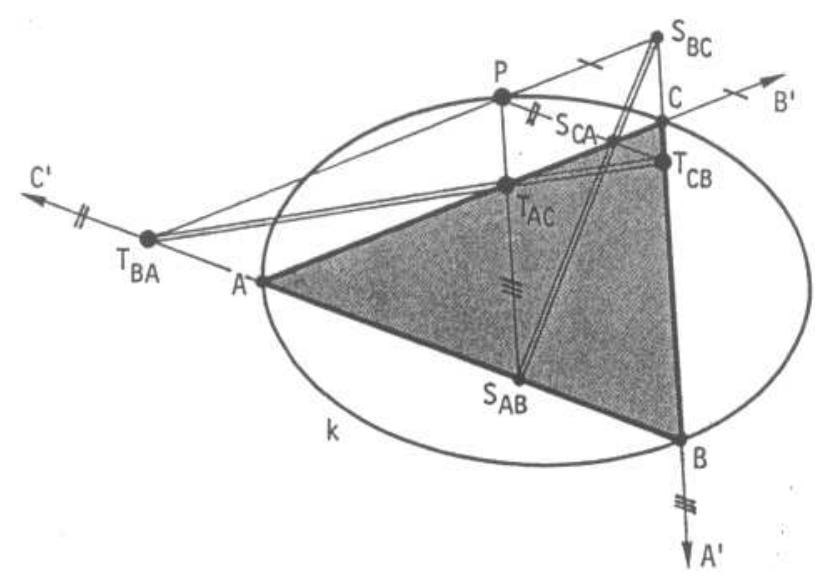

Abbildung 4 


$$
\text { "giering" — 2004/7/22 — 15:02 — page } 57 \text { — \#9 }
$$

Ist nur einer oder sind nur zwei der Leitpunkte $A^{\prime}, B^{\prime}, C^{\prime}$ Fernpunkte, dann läßt sich Abbildung 2 ebenfalls in leicht durchschaubarer Weise affingeometrisch interpretieren.

3.2. Die folgenden Abbildungen 5 bis 8 zeigen die Kubiken $k_{S}$ und $k_{T}$ in einem kartesischen $x y$-Koordinatensystem, in dem die Ecken des Dreiecks $A B C$ die Koordinaten besitzen: $A(a, 0), B(b, 0), C(0, c)$. Der laufende Punkt $P$ erhalte nun die Koordinaten $(\xi, \eta)$. Die Koordinaten der Leitpunkte $A^{\prime}, B^{\prime}, C^{\prime}$ seien

$$
\begin{aligned}
A^{\prime}([1-\alpha] b, \alpha c), B^{\prime}([1-\beta] a, \beta c), C^{\prime}([1-\gamma] a+\gamma b, 0) \\
\\
\operatorname{mit} \alpha \beta \gamma \neq 0, \alpha \neq 1, \beta \neq 1, \gamma \neq 1 .
\end{aligned}
$$

Als Kollinearitä̈sbedingung für die Punkte $S_{A B}, S_{B C}, S_{C A}$ und damit als Gleichung der Kubik $k_{S}$ erhält man nach einfacher Umformung:

$$
\left|\begin{array}{ccc}
{[1-\alpha] b \eta-\alpha c \xi} & 0 & \eta-\alpha c \\
{[1-\beta] b(c \xi+a \eta-a c)} & \beta c \xi-([1-\beta] a-b) \eta-\beta b c & c \xi+b \eta-c([1-\beta] a+\beta b) \\
a\{c \xi+([1-\gamma] a+\gamma b)(\eta-c)\} & \gamma(a-b) \eta & c \xi+a \eta-c([1-\gamma] a+\gamma b)
\end{array}\right|=0 .
$$

Also Kollinearitä̈sbedingung für die Punkte $T_{A C}, T_{C B}, T_{B A}$ und damit als Gleichung der Kubik $k_{T}$ folgt entsprechend:

$$
\left|\begin{array}{ccc}
{[1-\beta] a \eta-\beta c \xi} & 0 & \eta-\beta c \\
{[1-\alpha] a(c \xi+b \eta-b c)} & \alpha c \xi-([1-\alpha] b-a) \eta-\alpha a c & c \xi+a \eta-c([1-\alpha] b+\alpha a) \\
b\{c \xi+([1-\gamma] a+\gamma b)(\eta-c)\} & {[1-\gamma](b-a) \eta} & c \xi+b \eta-c([1-\gamma] a+\gamma b)
\end{array}\right|=0
$$

Abbildung 5 zeigt die Kubiken $k_{S}$ und $k_{T}$ zum Dreieck $A B C(a=3, b=9$, $c=6)$ mit den nicht kollinearen Leitpunkten $A^{\prime}, B^{\prime}, C^{\prime}\left(\alpha=\frac{1}{2}, \beta=\frac{1}{3}, \gamma=\frac{2}{3}\right)$ und den nicht kopunktalen Geraden $A A^{\prime}, B B^{\prime}, C C^{\prime}$. 
$\bigoplus$

"giering" — 2004/7/22 - 15:02 — page 58 - \#10

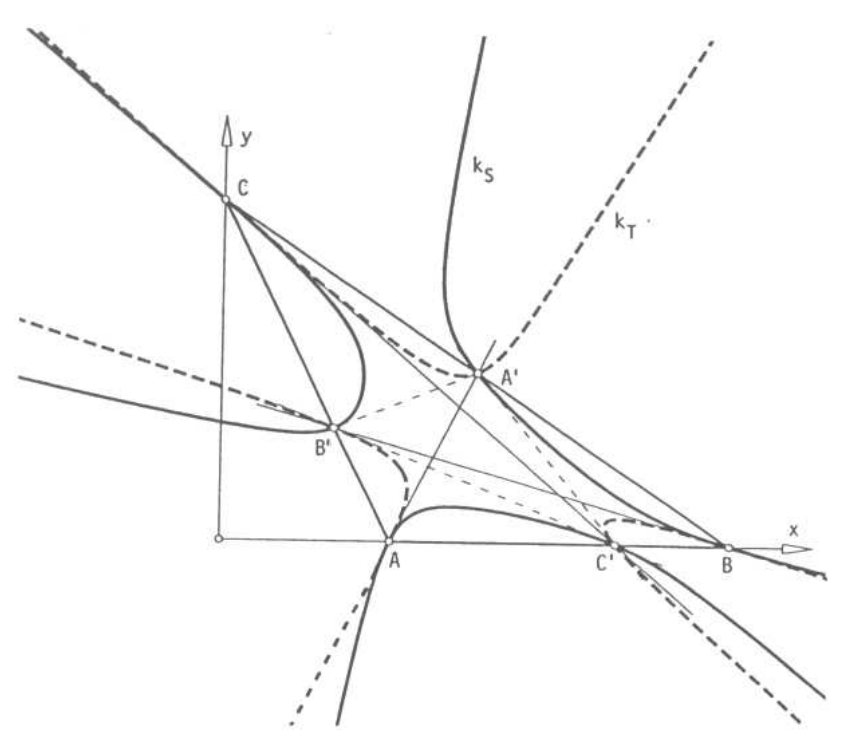

Abbildung 5

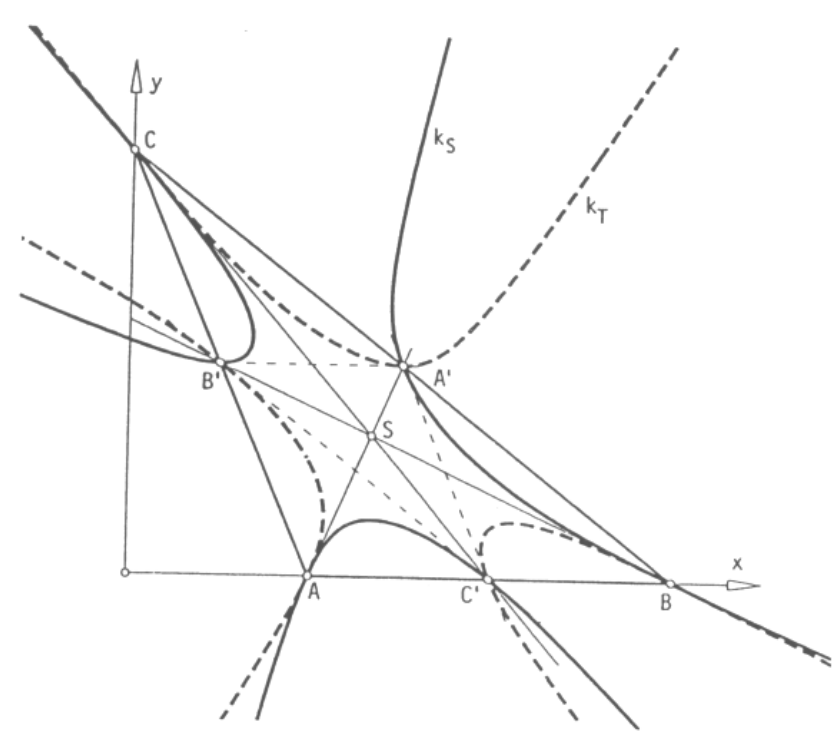

Abbildung 6 
Abbildung 6 zeigt die Kubiken $k_{S}$ und $k_{T}$ zum Dreieck $A B C(a=3, b=$ $9, c=7$ ) mit den Seitenmitten als Leitpunkten $A^{\prime}, B^{\prime}, C^{\prime}$ (affingeometrisches Beispiel). Die Geraden $A A^{\prime}, B B^{\prime}, C C^{\prime}$ sind die - im Schwerpunkt $S$ kopunktalen - Schwerlinien des Dreiecks $A B C$.

Abbildung 7 zeigt die Kubiken $k_{S}$ und $k_{T}$ zum Dreieck $A B C(a=3, b=9$, $c=6$ ) mit den Höhenfußpunkten als Leitpunkten $A^{\prime}, B^{\prime}, C^{\prime}$ (euklidischgeometrisches Beispiel). Die Geraden $A A^{\prime}, B B^{\prime}, C C^{\prime}$ sind die - im Höhenschnittpunkt $H$ kopunktalen - Höhen des Dreiecks $A B C$.

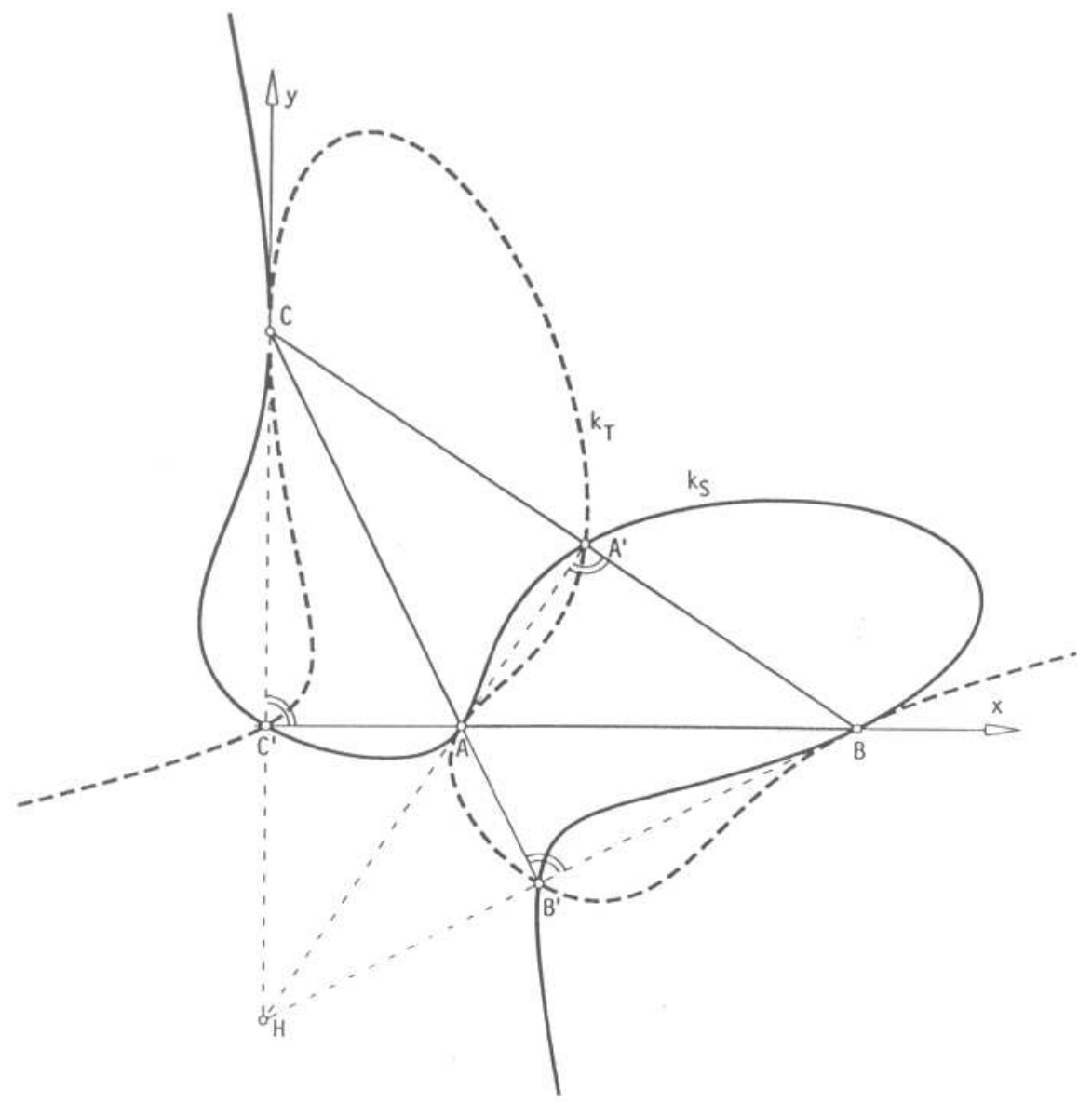

Abbildung 7 
Abbildung 8 zeigt die Kubiken $k_{S}$ und $k_{T}$ für ein gleichseitiges Dreieck $A B C$ mit den Höhenfußpunkten als Leitpunkten.

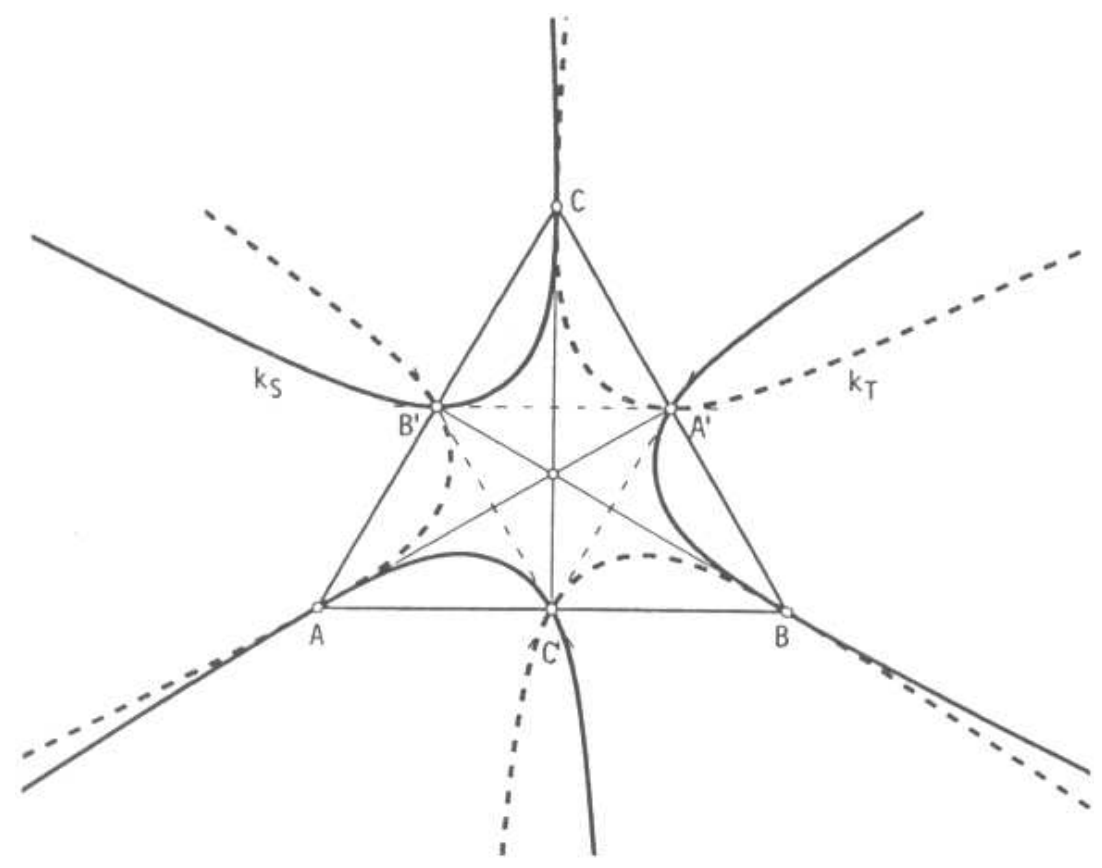

Abbildung 8

\section{Ausblick auf differentialgeometrische Fragen}

Durchläuft ein Punkt $P$ eine der Kubiken $k_{S}, k_{T}$ oder bei deren Zerfall einen der Kegelschnitte $k$ oder $k^{*}$, dann durchläuft jede der Geraden $S_{A B} S_{B C} S_{C A}$, $T_{A C} T_{C B} T_{B A}$ eine Geradenschar, die jeweils zur Bestimmung ihrer Hüllkurve einlädt. Wir bestimmen diese Hüllkurven in dem besonders einfachen Fall eines gleichseitigen Dreiecks $A B C$, in dem die Fernpunkte der Dreieckseiten als Leitpunkte $A^{\prime} \in B C, B^{\prime} \in C A, C^{\prime} \in A B$ gewählt werden. Dann stimmen die Kubiken $k_{S}, k_{T}$ nach Abschnitt 2.1 überein und zerfallen in die Leitpunktgerade (Ferngerade) und den Umkreis des Dreiecks $A B C$.

Abbildung 9 zeigt für einen beliebigen Umkreispunkt $P$ die Geraden $S_{A B} S_{B C} S_{C A}$ und $T_{A C} T_{C B} T_{B A}$, die ersichtlich einen Winkel von $60^{\circ}$ einschließen. Abbildung 9 


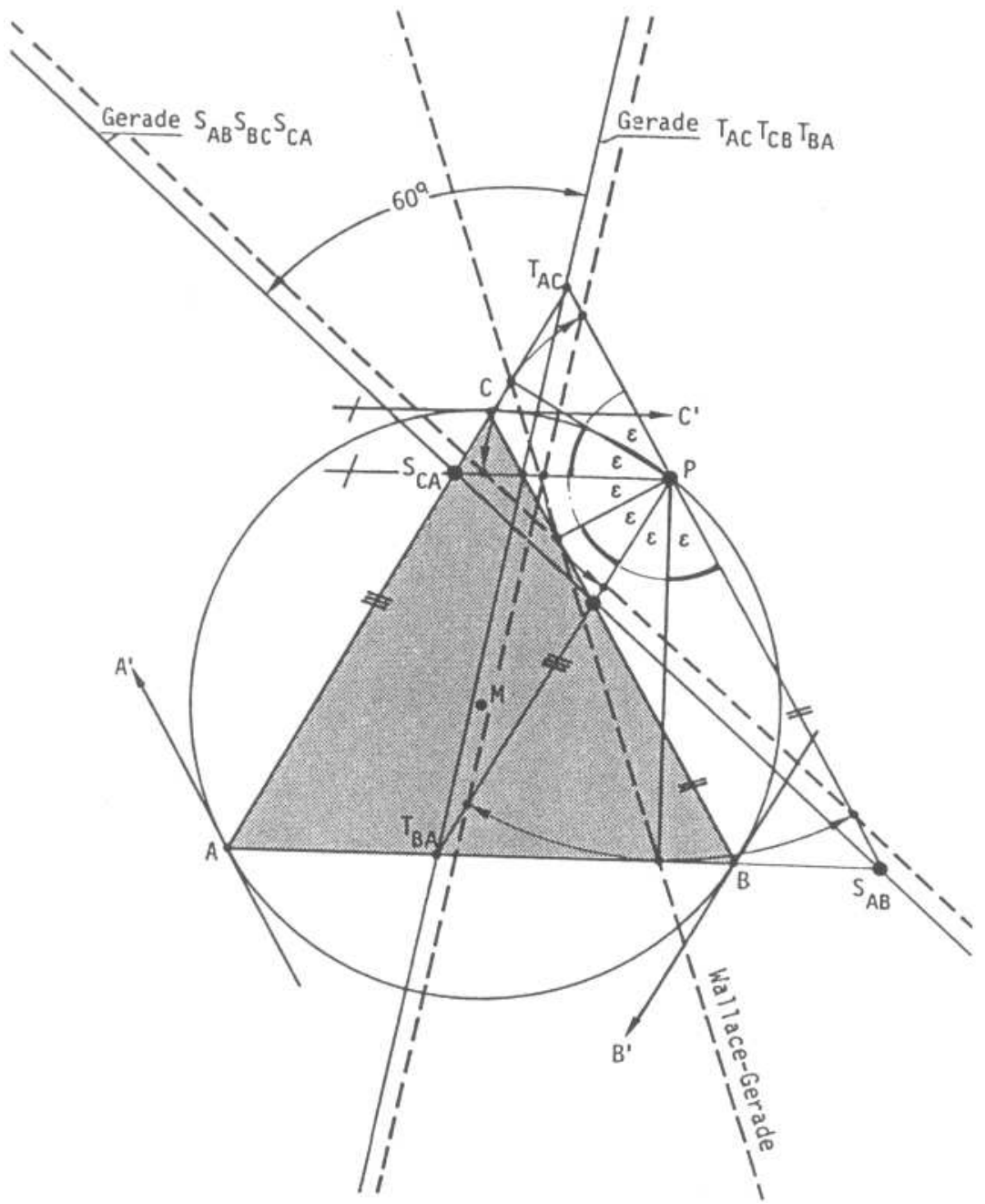

Abbildung 9

zeigt außerdem die Wallace-Gerade ${ }^{4}$ desselben Umkreispunktes $P$ zum Dreieck $A B C$. Dreht man die Wallace-Gerade um $P$ im Uhrzeigersinn (Gegenzeigersinn) durch $\varepsilon=30^{\circ}$, so ist ihre Drehlage parallel zur Geraden $T_{A C} T_{C B} T_{B A}$ (bzw.

${ }^{4}$ Fällt man von einem Punkt $P$ des Umkreises eines Dreiecks $A C$ die Lote auf die Dreieckseiten, so liegen die Lotfußpunkte kollinear, auf der Wallace-Geraden von $P$ zum Dreieck $A B C$. 
zu $\left.S_{A B} S_{B C} S_{C A}\right)$. Die Wallace-Gerade von $P$ schneidet daher jede der Geraden $T_{A C} T_{C B} T_{B A}$ und $S_{A B} S_{B C} S_{C A}$ unter $30^{\circ}$.

Abbildung 10 zeigt die Hüllkurven der Geraden $S_{A B} S_{B C} S_{C A}$ und $T_{A C} T_{C B} T_{B A}$, wenn der Punkt $P(\xi, \eta)$ den Umkreis des gleichseitigen Dreiecks $A B C$ mit den Ecken $A(-b, 0), B(b, 0), C(0, b \sqrt{3})$ durchläuft. Der Umkreis des Dreiecks $A B C$ besitzt den Mittelpunkt $M\left(0, \frac{b}{3} \sqrt{3}\right)$ und den Radius $\overline{M P}=\frac{2 b}{3} \sqrt{3}$.

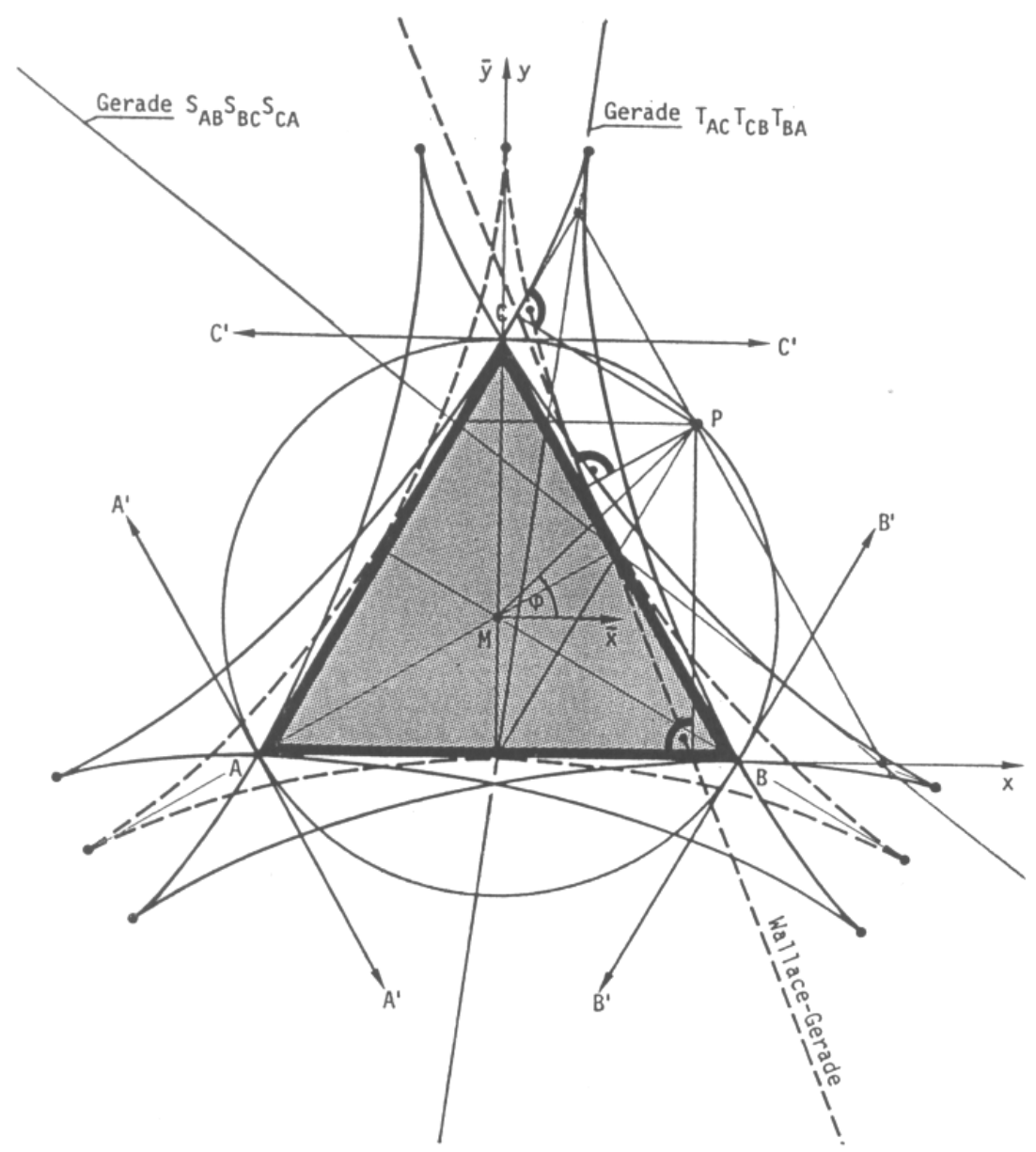

Abbildung 10 
Wählt man den Winkel $\varphi$ des Radius $\overline{M P}$ gegen die positive $x$-Achse als Parameter, so lautet die Darstellung des Umkreises

$$
\xi=\frac{2 b}{3} \sqrt{3} \cos \varphi, \quad \eta=\frac{b}{3} \sqrt{3}(1+2 \sin \varphi) \quad 0 \leq \varphi<2 \pi
$$

und die Punkte $S_{A B}$ und $S_{C A}$ erhalten die Koordinaten ${ }^{5}$

$$
\begin{gathered}
S_{A B}\left(\frac{b}{3}[1+2 \sin \varphi+2 \sqrt{3} \cos \varphi], 0\right), \\
S_{C A}\left(\frac{2 b}{3}[\sin \varphi-1], \frac{b}{3} \sqrt{3}[1+2 \sin \varphi]\right) .
\end{gathered}
$$

Legt man durch die Translation $x=\bar{x}, y=\bar{y}+\frac{b}{3} \sqrt{3}$ den Koordinatenursprung in den Umkreismittelpunkt $M$, dann erhält die Gerade $S_{A B} S_{B C} S_{C A}$ im $\bar{x} \bar{y}$ Koordinatensystem die Gleichung:

$$
(1+2 \sin \varphi) \bar{x}+(\sqrt{3}+2 \cos \varphi) \bar{y}=\frac{2 b}{3}(2 \sin \varphi+\sqrt{3} \sin 2 \varphi-\cos 2 \varphi)=: \Omega(\varphi) .
$$

Aus (16) und der nach $\varphi$ differenzierten Gleichung (16):

$$
\cos \varphi \bar{x}-\sin \varphi \bar{y}=\frac{2 b}{3}(\cos \varphi+\sqrt{3} \cos 2 \varphi+\sin 2 \varphi)=: \Lambda(\varphi)
$$

berechnet man als Parameterdarstellung der Hüllkurve der Geraden $S_{A B} S_{B C} S_{C A}$ :

$$
\begin{aligned}
& \bar{x}=\frac{\Omega(\varphi) \sin \varphi+\Lambda(\varphi)(\sqrt{3}+2 \cos \varphi)}{2+\sin \varphi+\sqrt{3} \cos \varphi} \\
& \bar{y}=\frac{\Omega(\varphi) \cos \varphi-\Lambda(\varphi)(1+2 \sin \varphi)}{2+\sin \varphi+\sqrt{3} \cos \varphi} .
\end{aligned}
$$

Die Dreieckseiten $A B, B C, C A$ sind spezielle Geraden $S_{A B} S_{B C} S_{C A}$. Aus den Gleichungen (18) folgt:

$A B$ berührt die Hüllkurve (18) in $B\left(\right.$ für $\left.\varphi=-30^{\circ}=-\frac{\pi}{6}\right)$,

$B C$ berührt die Hüllkurve (18) in $C$ (für $\left.\varphi=90^{\circ}=\frac{\pi}{2}\right)$ und

$C A$ berührt die Hüllkurve (18) in $A$ (für $\left.\varphi=210^{\circ}=\pi+\frac{\pi}{6}\right)$.

${ }^{5}$ Wir beschränken uns im Folgenden auf die Formeln zur Hüllkurve der Geraden $S_{A B} S_{B C} S_{C A}$.

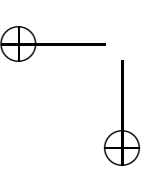




$$
\text { "giering" — 2004/7/22 — 15:02 — page } 64 \text { — \#16 }
$$

Das Dreieck $A B C$ ist also der Hüllkurve (18) derart einbeschrieben, daß $A B$ in $B, B C$ in $C$ und $C A$ in $A$ die Hüllkurve berühren.

Wendet man auf (18) die Parametertransformation $t=\tan \frac{\varphi}{2}$ an, so stellt sich für die Hüllkurve (18) - nach Kürzung mit dem Faktor $(t+[2+\sqrt{3}])^{2}-$ die gebrochen rationale Parameterdarstellung 4. Ordnung ein:

$$
\begin{aligned}
& \bar{x}=\frac{2 b}{3} \frac{(5-3 \sqrt{3}) t^{4}+2(2-\sqrt{3}) t\left(t^{2}-3 t+1\right)+(\sqrt{3}-1)}{(2-\sqrt{3})\left(t^{2}+1\right)^{2}} \\
& \bar{y}=\frac{2 b}{3} \frac{(2-\sqrt{3}) t^{4}+2 t^{3}+2(4 \sqrt{3}-7) t+(\sqrt{3}-2)}{(2-\sqrt{3})\left(t^{2}+1\right)^{2}} .
\end{aligned}
$$

Zusammenfassend gilt:

Satz 4. Durchläuft ein Punkt $P$ den Umkreis eines gleichseitigen Dreiecks $A B C$ und sind die Leitpunkte $A^{\prime} \in B C, B^{\prime} \in C A, C^{\prime} \in A B$ die Ferpunkte der Dreieckseiten, so ist die Hüllkurve der Geraden $S_{A B} S_{B C} S_{C A}$ (wie die Hüllkurve der Wallace-Geraden, siehe [5], S.856) eine geschlossene, 3-spitzige, dreiachsig symmetrische rationale Kurve 4. Ordnung (eine Steiner-Hypozykloide, siehe [7], S.175ff.; [8], S.68; [9], S.142).

Der Konstruktion der Geraden $S_{A B} S_{B C} S_{C A}$ und $T_{A C} T_{C B} T_{B A}$ entnimmt man unmittelbar: Durch Spiegelung einer Geraden $S_{A B} S_{B C} S_{C A}$ an $A M, B M$ oder $C M$ entsteht eine Gerade $T_{A C} T_{C B} T_{B A}$ und umgekehrt. Daraus folgt:

Satz 5. Die Hüllkurven der Geraden $S_{A B} S_{B C} S_{C A}$ und der Geraden $T_{A C} T_{C B} T_{B A}$ liegen zueinander symmetrisch bezüglich der Höhen des Dreiecks $A B C$ (Abbildung 10).

\section{Weiterer Ausblick}

Das in den Abschnitten 1.-4. vorgestellte Thema ist weiter ausbaufähig. Wir nennen dafür drei Beispiele.

(1) Eine eingehende Untersuchung der Hüllkurven der Geraden $S_{A B} S_{B C} S_{C A}$ und $T_{A C} T_{C B} T_{B A}$ (nicht nur beim gleichseitigen Dreieck mit Fernleitpunkten) auf ihre Gestalt und ihre gegenseitige Lage dürfte reizvoll sein.

(2) In Abbildung 2 ist die Bahn des Geradenschnittpunkts $S_{A B} S_{B C} S_{C A} \cap$ $T_{A C} T_{C B} T_{B A}$ von Interesse, wenn der Punkt $P$ den Kegelschnitt $k$ durchläuft. 


$$
\text { "giering" — 2004/7/22 — 15:02 — page 65 — \#17 }
$$

(3) Man kann der Frage nachgehen, für welche Punkte $P$ der Dreiecksebene die sechs Punkte $S_{A B}, S_{B C}, S_{C A}, T_{A C}, T_{C B}, T_{B A}$ auf einem Kegelschnitt liegen.

\section{Literatur}

[1] Z. Čerin, The Neuberg cubic in locus problems, Math. Pannonica 11 (2000), 109-124.

[2] O. Giering, Kopunktalitätsprobleme bei Dreiecken, Österr. Akad. Wiss., Sitzungsber. Abt. II 209 (2000), 3-18.

[3] B. Gilbert, The Lemoine cubic and its generalizations, Forum Geom. 2 (2000), 47-63.

[4] J. Lense, Analytische projektive Geometrie, R. Oldenbourg, München - Wien, 1965.

[5] J. Naas und H. L. Schmid, Mathematisches Wörterbuch, Band II, Akademie-Verlag Berlin und B. G. Teubner Stuttgart, 1972.

[6] G. M. Pinkernell, Cubic curves in the triangle plane, J. Geom. 55 (1996), 141-161.

[7] H. Schmidt, Ausgewählte höhere Kurven, Kesselringsche Verlagsbuchhandlung Wiesbaden, 1949.

[8] H. Schupp und H. Dabrock, Höhere Kurven, BI Wissenschaftsverlag, Mannheim Leipzig - Wien - Zürich, 1995.

[9] H. Wieleitner, Spezielle ebene Kurven, G. J. Göschen'sche Verlagshandlung, Leipzig, 1908.

OSWALD GIERING

ZENTRUM MATHEMATIK

TECHNISCHE UNIVERSITÄT MÜNCHEN

D-85748 GARCHING

GERMANY

(Received October, 2003) 